\title{
Creatividad publicitaria y audiovisual a través de la web: proyectos de formación aplicada en el seno de la universidad
}

\author{
Gloria JiMÉNEZ MARÍN \\ Universidad de Sevilla \\ gloria_jimenez@us.es \\ Rodrigo ELÍAS ZAMBRANO \\ Universidad de Huelva \\ rodrigoelias@us.es \\ Carmen Silva Robles \\ Universidad de Cádiz \\ carmen.silva@uca.es
}

Recibido: 30/07/2012

Aceptado: 22/10/2012

\begin{abstract}
Resumen
Nos encontramos ante un nuevo modo de consumir medios, ante una tecnología diferente. Estamos en la era del marketing interactivo, de la web 2.0 (y casi 3.0), donde la unión creatividad - tecnología es un par indisoluble. Así, durante varios cursos académicos se viene realizando en varias universidades andaluzas un proyecto piloto de creación de una web 2.0 como espacio de trabajo para crear e intercambiar ideas en el contexto de los estudios de Comunicación Audiovisual y Publicidad y RR.PP. Así, el aprendizaje basado en proyectos reales, uno de los principios del EEES, es el origen de esta iniciativa: la creación de un lugar de encuentro para la generación de ideas empresariales en el seno de la creatividad publicitaria y audiovisual.
\end{abstract}

Palabras clave: 2.0, Competencia, Comunicación, Enseñanza, Web.

\section{Advertising and Audiovisual Creativity athrough the Web: perational Training Projects Within the University}

\begin{abstract}
We are in a new way of consuming media, with a different technology. This is the internet era of interactive marketing, web 2.0 (almost 3.0), where the union creativity-technology is an inseparable pair. So, for several academic years, has been done in some Andalusian universities a pilot project to create a 2.0 webspaces to create and share ideas in the context of studies of Advertising and P.R. and Audiovisual creativity. Thus, the actual project-based learning, one of the principles of EEES, is the origin of this initiative: the creation of a meeting place for the generation of business ideas within an audiovisual and advertising creativity.
\end{abstract}

Keywords: 2 .0. Communication, Competence, Learning, Web.

\section{Referencia normalizada}

JIMÉNEZ MARÍN, Gloria; ELÍAS ZAMBRANO, Rodrigo y SILVA ROBLES, Carmen (2012): “Creatividad publicitaria y audiovisual a través de la web: proyectos de formación aplicada en el seno de la universidad". Estudios sobre el mensaje periodístico. Vol. 18, núm. especial noviembre, págs.: 551-558. Madrid, Servicio de Publicaciones de la Universidad Complutense.

Sumario: 1. Introducción. 2. Metodología. 3. Desarrollo; 3.1. La web 2.0; 3.2. Nuevas competencias y nuevos perfiles; 3.3. Nuevas tecnologías, nuevos modelos de educación. 4 Conclusiones. 5. Referencias bibliográficas 


\section{Introducción}

Tanto las nuevas metodologías por las que viene apostando el profesorado universitario, como las innovaciones docentes fomentadas por parte de los vicerrectorados de docencia, parten de una base común: el hecho de que los alumnos son el núcleo de la didáctica constituyéndose como los protagonistas teniendo, por tanto, responsabilidades, toma de decisiones, independencia. De este modo, el alumno gestiona sus recursos estableciendo una relación de consultoría con el profesor y de tutoría con los compañeros. Y, al mismo tiempo, el rol del profesor difiere: pasa de ser el protagonista absoluto, a ser un colaborador, un tutor.

En este sentido, el profesor deja de ser fuente de todo conocimiento y pasa a actuar como guía de los alumnos, facilitándoles el uso de los recursos y las herramientas que necesitan para explorar y elaborar nuevos conocimientos y destrezas; pasa a actuar como gestor de la pléyade de recursos de aprendizaje y a acentuar su papel de orientador y mediador (Salinas, 1998). La actividad propuesta de innovación docente en nuestras universidades está relacionada, sobre todo, con la incorporación de las TIC a los procesos de enseñanza aprendizaje.

\section{Metodología}

Aprovechamos el potencial de las TIC que la Universidad de Cádiz puso a nuestro alcance para, posteriormente, llevar el proyecto a otras universidades, como Universidad de Sevilla o Universidad de Huelva. Así, la organización de sistemas de enseñanza-aprendizaje en entornos virtuales supuso un proceso de innovación pedagógica basado en la creación de las condiciones para desarrollar la capacidad de aprender y adaptarse, tanto de las organizaciones como de los individuos, y desde esta perspectiva podemos entender la innovación como un proceso intencional y planeado, que se sustenta en la teoría y en la reflexión y que responde a las necesidades de transformación de la prácticas para un mejor logro de los objetivos (Fullan y Stiegelbauer, 1991; Rhodes, 1994; Kofman y Senge, 1995; Fullan, 2002).

En este sentido la labor del docente no sólo se limita a transmitir conocimiento, sino que se orienta a estimular el aprendizaje y ofrecer asesoramiento. La metodología, pues, es la puesta en práctica de conceptos y procedimientos expuestos en las clases teóricas mediante explicaciones y exposición de caso reales: concretamente la creación de una red social 2.0 como espacio y posible fuente de emprendizaje empresarial, para complementar la formación del alumnado. Del siguiente modo: en una primera fase, en lo que se refiere a la formación en las aulas, el proyecto plantea desarrollar el siguiente contenido:

- Explicación de la idea y presentación de la actividad.

- Invitación a equipos participantes

- Discusiones de grupo

- Visualización de ejemplos

A raíz de ahí, se conciertan conferencias en torno a la situación actual de las nuevas TIC aplicables al ámbito del Audiovisual y de la Publicidad y las RR.PP., oportunidades de trabajo, tendencias creativas en el mundo y la importancia de la nuevas 
tecnologías de la información y de la comunicación en la Publicidad y las RR.PP. Con un grupo de expertos en comunicación 2.0 (Facebook, Twitter, Tuenti, LinkedIn...) para protagonizar conferencias y un mesa redonda, para los alumnos bajo el tema 'Oportunidades de las redes sociales. La creatividad como forma para ser eficaz'. A partir del panel, la creación de una red social web 2.0 como fuente de conocimiento $\mathrm{y}$ de negocios futuro para, posteriormente, realizar una mesa redonda. Por último, la presentación de las ideas de los alumnos, con su correspondiente valoración por parte de un tribunal experto en marketing y comunicación interactiva, seguido de unas conclusiones por parte de los alumnos de la experiencia.

En este sentido, ¿cuáles son los objetivos perseguidos? Conocemos que las nuevas ideas y concepciones sobre la enseñanza parten del hecho de que el alumno pasa a ser el centro del proceso didáctico, pasa de ser un sujeto pasivo en el aula a ser un sujeto implicado en el proceso de aprendizaje, con una serie de responsabilidades sobre la toma de decisiones. El estudiante se hace partícipe de sus propios recursos y establece relaciones de ayuda con el profesor y con los propios compañeros de aula. El papel del profesor también varía; de ser el sujeto protagonista asume un nuevo papel como colaborador en el proceso de la enseñanza. Por ello, el objetivo principal es la puesta en práctica del aprendizaje basado en un proyecto real que cumpla dos objetivos: creación de una idea de negocio, por un lado, y cumplimentar la formación en materia publicitaria, por otro. Todo ello a través de la puesta en práctica de una red social web 2.0.

Consideramos que esta forma de trabajo, representa una alternativa congruente con el modelo del rediseño de la práctica docente donde nuevas concepciones sobre la enseñanza sustentan el hecho de que el alumno pasa de ser un sujeto pasivo a ser un sujeto implicado en el proceso de aprendizaje, siendo responsable de sus propios recursos. El papel del profesor también varía: de ser el sujeto protagonista asume un nuevo papel como colaborador en el proceso de la enseñanza.

La aplicación de estos principios a la enseñanza en creatividad publicitaria en cualquiera de sus formas (audiovisual, impresa, etc.) se ha ido conjugando además con algunas estrategias de aprendizaje propias del ejercicio de la profesión publicitaria. Entre ellas está la utilización del Rol-play, una simulación donde se establecen relaciones contractuales entre alumno-profesor para el diseño de campañas publicitarias desde su origen hasta su aprobación posterior. Estas estrategias se han aplicado con mayor frecuencia en los últimos cursos de la Licenciatura, llegando a evaluar a los estudiantes a través de presentaciones reales de campañas donde el anunciante está en muchas ocasiones presente. Y todo ello para dar paso a los estudios de Grado que ya se están implantando en la mayoría de las universidades españolas.

\section{Desarrollo}

\subsection{La web 2.0.}

Para entender de donde viene el término de Web 2.0 tenemos que remontarnos al momento en que Dale Dougherty utilizó este término en una conferencia en la que compartió una lluvia de ideas junto a Craig Cline. En dicho evento se hablaba del renacimiento y evolución de la web. El verdadero artífice de la expresión Web 2.0 es Tim O’Reilly (Celaya, 2008; Pisani y Piotet, 2008; Beelen, 2006; Cobo y Pardo, 2007), 
aunque es Celaya (2008: 27) quien sitúa en el año 2001 sus primeras anotaciones sobre Web 2.0. Por su parte, el resto de autores sitúan este acontecimiento entre 2004 y 2005.

Fue precisamente en septiembre de 2005 cuando O'Reilly publica la que se ha considerado como la primera y mayor referencia bibliográfica sobre el término, el artículo What Is Web 2.0. Design Patterns and Business Models for the Next Generation of Software. Según O'Reilly, siete son los principios constitutivos de las aplicaciones Web 2.0 (Pardo, 2007: 27 y 37): la Web como plataforma; el aprovechamiento de la inteligencia colectiva; la gestión de la base de datos como competencia básica; el fin del ciclo de las actualizaciones de versiones del software; los modelos de programación ligera junto a la búsqueda de la simplicidad; el software no limitado a un solo dispositivo; y las experiencias enriquecedoras de los usuarios.

Celaya la describe como una Web colaborativa que permite a sus usuarios acceder y participar en la creación de un conocimiento ilimitado, y como consecuencia de esta interacción se generan nuevas oportunidades de negocio para las empresas. En comparación con la Web 1.0 la caracteriza como webs más dinámicas y ricas en contenido multimedia (sonido, vídeo, metadatos, etc.). Una etapa en la que las empresas entienden que deben ofrecer a sus clientes y potenciales la posibilidad de comentar, valorar u opinar sobre los contenidos publicados con el fin de enriquecer la comunicación virtual entre ambas partes.

Son muchas las investigaciones y los estudios que en la actualidad pretenden teorizar y describir esto que ya ha llegado a ser considerado como un fenómeno social. Pisani y Piotet (2008: 45) afirman que estamos ante un fenómeno diferente en el que el comportamiento de los jóvenes en internet crea grandes tendencias. Éstas son las que generalizarán cuando crezcan, entren el mundo laboral e integren en su trabajo su manera actual de utilizar la web.. Lo que ellos denominan dinámica relacional puede describirse en los siguientes términos (Pisani y Piotet, 2008: 45):

- Las tecnologías están presentes, pero los usos y los hábitos de los usuarios son los protagonistas.

- La plataforma es realmente flexible, y permite que los usuarios la dominen con facilidad.

- Permite la posibilidad de comunicar en la nube, de manera libre.

- Es un espacio social y relacional que da la palabra a los aficionados expertos.

El concepto de Web 2.0 viene irremediablemente ligado al de Sociedad del Conocimiento. Castells (2002: 13) la define como "un nuevo paradigma tecnológico", que tiene dos expresiones fundamentales: Internet y la capacidad de recodificar la materia viva. Destaca además la importancia decisiva de esta dimensión cultural en la producción y las formas de las tecnologías que la han hecho posible, y analiza sus distintas capas: la universitaria, la hacker, las formas culturales alternativas y, finalmente, la cultura empresarial. La UNESCO (2005) ya expresaba que la sociedad del conocimiento es inseparable de los estudios sobre la sociedad de la información. Una Sociedad del Conocimiento es una sociedad con capacidad para generar, apropiar, y utilizar el conocimiento para atender las necesidades de su desarrollo y así construir su propio futuro, convirtiendo la creación y trasferencia del conocimiento en herramienta de la 
sociedad para su propio beneficio. En la misma línea que Castell se encuentra Khan (exsubdirector general de la UNESCO para la Comunicación y la Información) quien expresaba que "la sociedad de la Información es la piedra angular de las sociedades del conocimiento. [...] relacionado con la idea de la "innovación tecnológica" (2003: 27).

La web 2.0 también ha provocado cambios sustanciales en la forma de organizar la información disponible. Los nuevos medios de comunicación que han surgido en la Red no sólo han renunciado a producir específicamente para su público a favor de que sean éstos quienes lo hagan sino que además establecen sistemas para que sean también los usuarios quienes, de forma automática a través de sus actos, decidan sobre la jerarquización y la relevancia de los contenidos. Este fenómeno se conoce con el nombre de folksonomías, taxonomías construidas entre pares, y se materializa en la posibilidad de 'etiquetar' los contenidos para crear filtros colectivos de criterio social.

El término con el que se denomina el acto de participar en la generación de los contenidos de los sitios web 2.0 es el de User Generated Content. El UGC se refiere a los sistemas de colaboración donde el usuario no se limita a navegar entre las informaciones producidas por otros sino que se convierte él mismo en productor.

Mao (2007) habla del término Sharismo para referirse a la cultura participativa, un estado mental neuropsicológico que lleva a compartir conocimientos motivados por las características tecnológicas de la web 2.0 y las prácticas culturales que sostienen movimientos como el de Creative Commons y las comunidades de software libre. El sharismo es una propiedad innata de nuestro cerebro, abierto a establecer relaciones neuronales y compartir información en procesos creativos de producción de conocimiento. Así, "cuanto más abiertos y fuertemente conectadas estén nuestras neuronas, mejor será el entorno colaborativo para todo el mundo" (Mao, 2007).

En línea con dicho término encontramos otro que se ha convertido en todo un fenómeno: el crowdsourcing. Para entender plenamente su significado debemos comenzar definiendo el término del que surge: el outsourcing, que se refiere a la subcontratación de terceras empresas o individuos para externalizar parte del trabajo de su cadena productiva.

\subsection{Nuevas competencias y nuevos perfiles}

La web 2.0 tiene pues unas características concretas y específicas de recepción que la hacen, cuanto menos, diferente a las websites de la era 1.9 y versiones anteriores. Los conceptos 'creatividad', 'tecnología', 'interacción', 'simultaneidad', etc. son términos que conviven en esta nueva etapa de la red... Pero, en esta avenencia, el profesional de comunicación debe reinventarse, re educarse en este nuevo entorno para poder satisfacer así a empresas y sus marcas con propuestas atractivas y eficaces. Y, sobre todo, eficientes para, de este modo, poder explicarle a su cliente cómo puede llegar a su target y qué puede hacer con ellos. Todo ello mediante estrategias bien pensadas para cada público y capaz de diversificarse. En definitiva, cómo trazar la estrategia comunicativa a partir de las posibilidades que permite la web 2.0, donde existe una cultura participativa que marca el trabajo de gestión de la comunicación en la red y un concepto que nos ayuda a dilucidar cuáles son las competencias que deben desarrollar sus profesionales en estos entorno. 
Tapscotty y Williams aportan algunas ideas sobre transformaciones que abarcan incluso el aspecto económico: una nueva economía impulsada por el cambio tecnológico, la demografía y la globalización, que motoriza el cambio y la innovación basados en nuevas formas de colaboración. Es la web colaborativa (término que, aunque aún no está reconocido en la RAE, ya se plantea para su aceptación en próximas ediciones). Esta nueva realidad económica la expresan en base a cuatro principios básicos:

- Apertura

- Interacción entre iguales

- Compartir

- Actuación global

En el recorrido vivido por los alumnos desde el planteamiento original del problema hasta su solución, trabajan en equipo, compartiendo en esa experiencia de aprendizaje la posibilidad de practicar y desarrollar habilidades y competencias genéricas de carácter transversal, y de observar y reflexionar sobre actitudes y valores que con un método tradicional se podrían en acción. De este modo, el alumno toma responsabilidades y deciden soluciones básicas en su

\subsection{Nuevas tecnologías, nuevos modelos de educación}

Para Pardo (2007) la educación ha sido una de las disciplinas más beneficiadas con la irrupción de las nuevas tecnologías, especialmente las relacionadas a la Web 2.0. Por ello, resulta fundamental conocer y aprovechar la batería de nuevos dispositivos digitales, que abren inexploradas potencialidades a la educación y la investigación. Incluso en el argot académico algunos ya hablan del Aprendizaje 2.0. Al respecto plantea Piscitelli, que hoy día Internet no es tan solo un nuevo medio, sino un espacio virtual en el que pasan cosas. Se trata, más bien, de un territorio potencial de colaboración en el cual pueden desplegarse de manera adecuada procesos de enseñanza y aprendizaje (Piscitelli, 2005). Las aplicaciones Web 2.0 es la simplificación de la lectura y escritura en línea de los estudiantes. Esto se traduce en dos acciones sustantivas del proceso de aprendizaje: generar contenidos y compartirlos. Podría plantearse que este Aprendizaje 2.0 se apoya en dos principios básicos de la actual Web: contenidos generados por el usuario y arquitectura de la participación.

Bajo este panorama se consolida una Web que permite innovar hacia un modelo más rico en herramientas, espacios de intercambio y pedagógicamente más prolíficos, donde estudiantes y docentes pueden aportar sus conocimientos. Así, a principios de los noventa, Gibbons, Limoges, Nowotny, Schwartzman, Scott y Trow (1994) ya analizaban la idea de un conocimiento socialmente distribuido, potenciado por el uso de aplicaciones que favorecen el acceso, el intercambio de información y la generación colectiva de contenidos. Es decir, un conocimiento intercambiable, acumulativo, colaborativo, que puede ser compartido, transferido y convertido en un bien público.

Johnson (1992), por su parte, contribuye con que el conocimiento siempre se genera bajo una continua negociación y no será producido hasta que los intereses de varios actores estén incluidos. Este autor propone cuatro tipologías diferentes de aprendizaje: 
- Aprender haciendo (learning-by-doing).

- Aprender interactuando (learning-by-interacting).

- Aprender buscando (learning-by-searching).

- Aprender compartiendo (learning-by-sharing).

Gutiérrez (2003) define la alfabetización digital desde su dimensión de 'funcional', como la preparación básica para que cada persona pueda desenvolverse como tal en su entorno; y, por otra parte admite la creación de un nuevo modelo de sociedad con la significativa presencia de las tecnologías de la información y la comunicación. Desde esta perspectiva, Gutiérrez aboga por hablar de "alfabetización múltiple o multimedia" (2003: 12), entendida como la capacitación básica para comprender y expresarse con distintos lenguajes y medios. En su obra La alfabetización digital es algo más que ratones y teclas expone que la preparación básica para vivir en la Sociedad de la Información (alfabetización digital) va más allá de proporcionar las destrezas necesarias para el manejo de las actuales tecnologías. Este aprendizaje que denomina la alfabetización instrumental y que va ligada a las tecnologías de cada momento concreto, habría que completarlo con una alfabetización crítico-reflexiva que trascienda lo puramente tecnológico e instrumental para abordar la importancia de la información y la comunicación en sociedad digital. Estos conocimientos básicos sobre la trascendencia social, económica y cultural de los desarrollos tecnológicos serán válidos en 10 y 20 años si se actualizan debidamente. En este contexto de alfabetización digital Lara (2010) propone cuatro claves de trabajo: aprender con la red, sobre la red, en la red, para la red.

En el informe La educación mediática en la escuela 2.0, sus autores destacan la necesidad de integrar una metodología 2.0, es decir, se requiere la readaptación sustancial de los roles del profesor y del alumno, el modelo comunicativo, la teoría de aprendizaje, la introducción de Internet en la escuela, los recursos didácticos y herramientas web 2.0, y su uso para mejorar o favorecer la inclusión del alumnado con necesidades específicas de apoyo educativo.

\section{Conclusiones}

El proyecto en cuestión ha traído consigo, edición tras edición, el incremento de la capacidad analítica y crítica del alumno, en orden a favorecer la toma de decisiones y en el desarrollo de habilidades para el trabajo en equipo, y la búsqueda de información. En este sentido, es destacable también la valoración, por parte del alumno, del significado y el alcance del sistema empleado, así como el desarrollo de su potencial en el campo de la creación y de la gestión adecuarse a la realidad en las empresas y en las instituciones.

Es importante destacar la facilidad de trabajo con el alumnado dado que, como expresan algunos de los autores citados a lo largo del texto, es un lenguaje que permite, de muchos modos, aprender compartiendo; permite realizar un sistema colaborativo donde el egoísmo informacional desaparece, la creatividad aumenta y, con todo ello, la productividad es mayor y el nivel de satisfacción a la finalización del trabajo es plenamente satisfactoria tanto para alumnado como para profesorado. Y en la puesta en práctica, también en el ámbito empresarial. 


\section{Referencias bibliográficas}

CASTELLS, Manuel (2002): La era de la información, economía, sociedad y cultura. Vol. 1: La sociedad en red. Madrid, Siglo XXI.

CELAYA, Javier (2008): La empresa en la Web 2.0. Barcelona, Gestión 2000.

COBO ROMANÍ, Cristóbal y PARDO KUKLINSKI, Hugo (2007): Planeta Web 2.0. Inteligencia colectiva o medios fast food. Grup de Recerca d'Interaccions Digitals, Universitat de Vic. Flacso Barcelona.

DOUGHERTY, Dale (2011): "What is web 2.0". En: http://oreilly.com/web2 /archive/what-is-web-20.html. [Fecha de consulta: 3/09/2012].

GIBBONS, Michael; LIMOGES, Camille; NOWOTNY, Helga; SCHWARTZAN, Simon; SCOTT, Peter; y TROW, Martin (1994): The new production of knowledge: The dynamics of science and research in contemporary societies. London, Sage.

GUTIÉRREZ MARTÍN, Alfonso (2003): Alfabetización digital. Algo más que ratones y teclas. Buenos Aires. Gedisa Editora.

LARA, Tíscar (2010): "Educación y Redes Sociales". Documentación entregada en el curso de verano Ser y Estar en Internet: educar con y sobre las redes sociales, ofrecido por la Universidad Internacional de Andalucía, en su sede de Málaga del 14 al 16 de julio de 2010.

MAO, Isaac (2007): “Sharism: A Mind Revolution”. En http://freesouls.cc/essays/07isaac-mao-sharism.html. [Fecha de consulta: 12/07/2012].

O’REILLY, Tim (2006): "Qué es Web 2.0. Patrones del diseño y modelos del negocio para la siguiente generación del software". En: http://sociedadinformacion.fundacion.telefonica.com/DYC/SHI/seccion=1188\&idioma=es_ES\&id=20091001163 00061\&activo $=4$. do?elem $=2146 \#$. [Fecha de consulta: $23 / 07 / 2012$ ]

PISANI, Francis y PIOTET, Dominique (2008): La Alquimia de las Multitudes. Barcelona. Paidós

PISTICELLI, Alejandro (2005): “Tecnologías educativas. Una letanía sin ton ni son”. En Revista de Estudios Sociales, 22: http://res.uniandes.edu.co/pdf/descargar.php ?f=./data/Revista_No_22/13_Debate3.pdf. [Fecha de consulta: 23/05/2012].

SALINAS IBÁNEZ, Jesús (1998): "Redes y Educación: Tendencias en educación flexible y a distancia", En PÉREZ, R. y otros: Educación y tecnologías de la educación. II Congreso Internacional de Comunicación, tecnología y educación. Oviedo.

UNESCO (2003): Towards Knowledge Societies. An Interview with Abdul Waheed Khan, World of Science Vol. 1, No. 4 July-September 2003, UNESCO's Natural Sciences Sector. 


\section{Gloria JIMÉNEZ MARÍN}

Universidad de Sevilla - Universitat Oberta de Catalunya gloria_jimenez@us.es

Facultad de Comunicación, Despacho K3-Avda. Américo Vespuccio, s/n - 41092 - Sevilla Doctora por la Universidad de Sevilla y Profesora Contratada Dra. en el Departamento de Comunicación Audiovisual y Publicidad y Literatura de la misma universidad en el área de publicidad y RR.PP. Licenciada en Periodismo y Licenciada en Publicidad y RR.PP., colabora con la Universitat Oberta de Catalunya y es Máster en diseño publicitario. Tras su paso por la actividad profesional en agencias de publicidad, su actividad investigadora se centra las relaciones entre la publicidad y el arte, y la publicidad y el cine, así como el estudio de las acciones comunicativas y de merchandising en los espacios comerciales. Es autora de artículos científicos y divulgativos, libros y capítulos, además de participante en congresos universitarios y profesionales.

\section{Rodrigo ELÍAS ZAMBRANO}

Universidad de Huelva - Universidad de Sevilla rodrigoelias@us.es

Facultad de Comunicación, Despacho K3-Avda. Américo Vespuccio, s/n - 41092 - Sevilla Licenciado en Comunicación Audiovisual por la Universidad de Sevilla y Máster en Gestión de Empresas AV por US, así como Experto en E-Learning, se encuentra realizando en la actualidad el Máster oficial en Comunicación y Educación AV en la Universidad de Huelva. Es docente en asignaturas relacionadas con el ámbito audiovisual y su relación con la publicidad, las nuevas tecnologías y la educomunicación. Es miembro del grupo de investigación SEJ420 y se encuentra preparando su tesis doctoral, centrada en la influencia de la televisión en la educación para adultos. Ha publicado artículos y capítulos de libros sobre cine y publicidad, educomunicación y sobre la relación audiovisual - publicidad. A nivel profesional, está ligado al mundo de la realización AV desde 1999 trabajando en informativos, retransmisiones deportivas y programas de distinta temática para Tele5, Antena3 y Canal Sur TV. También ha sido realizador para la productora de TV I.N.S. (Instant news services) en Bruselas con servicios a la RAI, la CNBC, Al Jazeera o NHK. Actualmente, compatibiliza sus tareas de realizador audiovisual con las de docente.

\section{Carmen SILVA ROBLES}

Universidad de Cádiz - Universitat Oberta de Catalunya

carmen.silva@uca.es

Facultad de Ciencias Sociales y de Comunicación, Avda. de la Universidad s/n - Edificio de usos múltiples. Despacho 2.10. Universidad de Cádiz. 11405 Jerez de la Frontera (Cádiz)

Profesora en el Departamento de Marketing y Comunicación de la Universidad de Cádiz es colaboradora en la Licenciatura de Publicidad y Relaciones Públicas en la Universitat Oberta de Catalunya. Directora de comunicación de la Organización Internacional de Ceremonial y Protocolo y docente habitual en los cursos de formación de la Confederación de Empresarios de Andalucía, en materia relacionada con la gestión de la publicidad y de las RR.PP. La Sra. Silva posee más de 10 años de experiencia en comunicación y combina su actividad docente e investigadora con la gestión de la agencia AO Consultancy. Actualmente se encuentra ultimando su tesis doctoral Proyecto de tesis Community Managers o gestores de comunidad: la dirección de relaciones públicas en la red. 